\title{
Komplikationen nach proximalen Femurfrakturen
}

\author{
Christoph Josten, Andreas Heinrich Tiemann
}

\section{Zusammenfassung}

Die in Deutschland mit jährlich 90000 Fällen auftretenden proximalen Femurfrakturen sind die häufigsten Knochenbrüche des alten Menschen. Die altersbedingt hohe Morbidität dieses Klientels führt zu besonderen Anforderungen bei der Versorgung derartiger Läsionen einerseits, hat aber auch das gehäufte Auftreten von Komplikationen zur Folge. Dabei werden Frühkomplikationen (innerhalb der ersten 3 postoperativen Monate) von Spätkomplikationen (nach 3 Monaten) un- terschieden. Haupsächlich treten in der postoperativen Frühphase thromboembolische Komplikationen auf, gefolgt von lokalen Problemen. Das Gros der Spätkomplikationen stellen Pseudarthrosen und Femurkopfnekrosen dar. Derartige Veränderungen führen nahezu zwingend zu erneuten Operationen, s.g. „Salvage Procedures“. Unstrittig ist dabei, dass nur ein sich an der Knochenbiologie, der Biomechanik und insbesondere am Allgemeinzustand des Patienten orientierendes, stringentes Therapiekonzept in diesen Fällen zum Erfolg führen kann.

\section{Einleitung}

Die proximale, hüftgelenksnahe Fraktur stellt heutzutage die häufigste Verletzung des älteren Menschen dar. Jährlich treten derartige Verletzungen in Deutschland z.Z. 90000-mal auf und nach Angaben des statistischen Bundesamtes von 1998 ist mit einer Zunahme von 50\% innerhalb der ersten Jahre des 21. Jahrhunderts zu rechnen [11]. Im Jahr 2030 wird der Anteil der über 60-jährigen in Deutschland voraussichtlich bei 24.5 Mio liegen (Gesamtbevölkerung dann $80 \mathrm{Mio}$ ). Die Wiederherstellung eines funktionierenden Hüftgelenkes sowie eines belastbaren Oberschenkels mit dem Ziel der frühestmöglichen Mobilisation der Verletzten und maximaler Unabhängigkeit sind oberste Maxime bei der Therapie proximaler Femurfrakturen. Dieses ist nur durch eine rasche und zielgerichtete Stabilisierung der Fraktur einerseits ebenso wie durch die konsequente Behandlung evtl. auftretender Komplikationen mög-

OP-JOURNAL 2002; 17: 148-153

(c) Georg Thieme Verlag Stuttgart · New York lich. Die Ursachen von Komplikationen sind mannigfaltig und sowohl in der Biologie der Betroffenen ( fortgeschrittenen Osteoporose, reduzierter Allgemeinzustand etc.) als auch in der Osteosynthese selbst zu suchen.

\section{Einteilung}

Grundsätzlich sind folgende Komplikationen nach der operativen Versorgung proximaler Femurfrakturen zu beachten:

Frühphase ( $<3$ Monate postoperativ)

Lokale Komplikationen, wie z.B. Wundheilungsstörungen oder Infektionen

- Systemische Komplikationen, wie z. B. thromboembolische Geschehen

- Komplikationen aufgrund technischer oder taktischer Fehler

„Spätere“ Phase (> 3 Monate postoperativ)

- Komplikationen aufgrund unzureichender „Knochenbiologie“, wie z. B. ausbleibende Frakturheilung mit Pseudarthrosenbildung oder Dislokation des Implantates bei schwerer Osteoporose.
Frühphase (<3 Monate postoperativ)

Die Komplikationsrate nach operativer Versorgung hüftgelenksnaher Frakturen ist hoch [22]. In einer Studie von 1998 konnten Vajanto et al. eine Gesamtkomplikationsrate von 38\% nach operativer Behandlung proximaler Femurfrakturen nachweisen. Es zeigte sich, dass die Komplikationsrate nach Stabilisierung von Schenkelhalsfrakturen deutlich über der nach Stabilisation pertrochantärer Frakturen lag [28]. Die häufigste systemische Komplikation stellte die Thromboembolie dar. Dementsprechend ist eine konsequente Thromboseprophylaxe mit niedermolekularem Heparin unabdingbar.

Lokale Komplikationen entstanden in 19,8\% der Fälle, wobei es sich im Wesentlichen um Therapieversager (6,58\%), Implantatlockerungen $(2,99 \%)$ und Femurkopfnekrosen $(2,09 \%)$ handelte. Lokale Wundprobleme in Form von oberflächlichen oder tiefen Infekten oder Hämatomen traten in jeweils $1,79 \%$ auf [28]. In diesem Zusammenhang sei auf die Bedeutung der intraoperativen Antibiotikaprophylaxe hingewiesen [12].

Die Mortalität lag bei $8 \%$ und somit auf dem aus der Literatur bekannten Niveau $[13,16,28]$.

In ihrer Studie von 2002 konnten Dzupa u. Mitarb. zeigen, dass die Mortalitätsrate bei Männern über 80 Jahre in der Risikogruppe ASA4 und höher signifikant erhöht war und dass die höchste Mortalität innerhalb dieser Gruppe innerhalb der ersten 3 Monate postoperativ auftrat [7]. In ihrer Arbeit von 1999 konnten Smektala et al. intraoperative Komplikationen nur in 1,7\% der Fälle nachweisen. Die Gesamthäufigkeit lag bei 26,1\%. Interessanterweise sank über einen Beobachtungszeitraum von 5 Jahren der Anteil von kardiovaskulären Komplikationen von $7,7 \%$ in 1993 auf 5,5\% in 1997. Im gleichen Zeitraum hingegen stieg der Anteil 

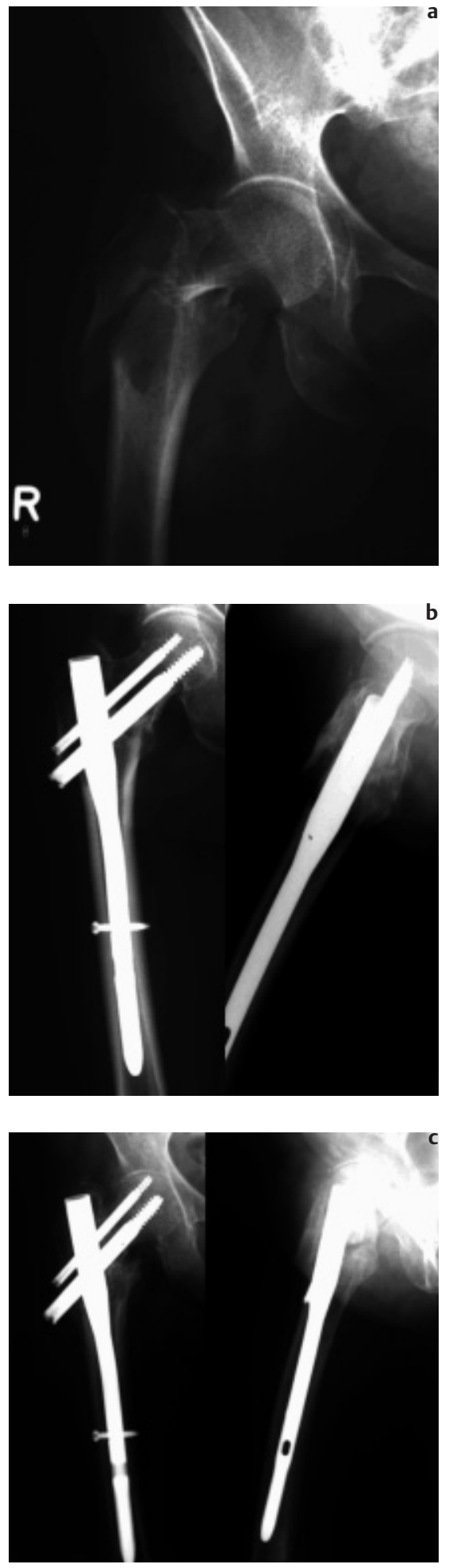
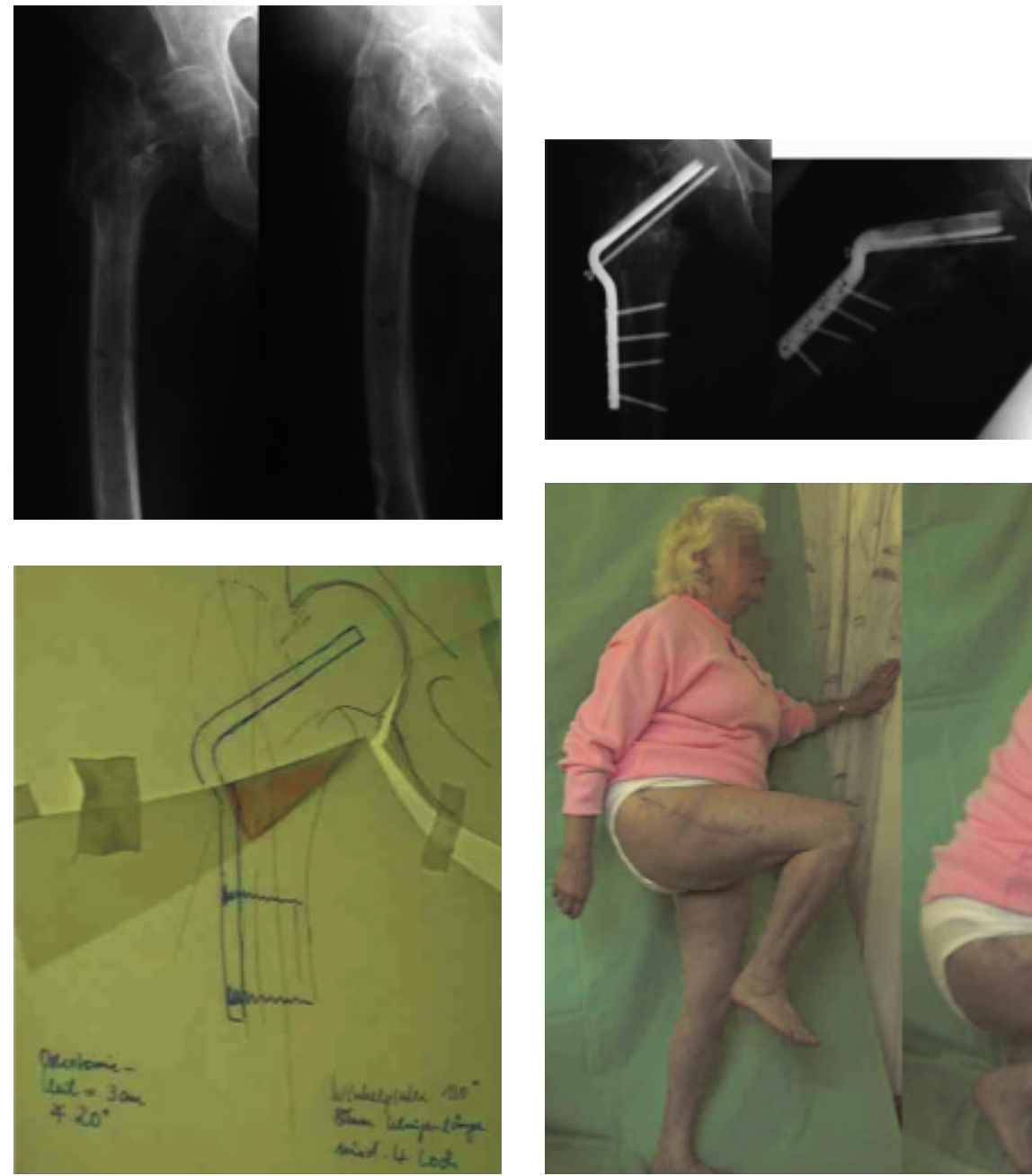

Abb.1 (a) 70-jährige Patientin mit pertochantärer Oberschenkelfraktur. (b) Primärversorgung mit PFN. (c) Zustand des PFN nach 4 Monaten. (d) Varusfehlstellung 2 Wochen nach vorzeitiger Metallentfernung. (e) Planung der Korrekturosteotomie. (f) Korrekturosteotomie mittels $135^{\circ}$ Klingenplatte 7 Monate nach Unfall. (g) klinisches Bild 12 Monate nach Unfall.

von Wundheilungsstörungen von $4.9 \%$ auf $6,0 \%$ (Tab.1) [24].

\section{— „Spätere“ Phase}

Wesentliche Ursache für Spätkomplikationen sind Pseudarthrosenbildung und Femurkopfnekrosen (Tab.2) [22]. In derartigen Fällen ist zumeist eine erneute Stabilisierung angezeigt.

Über Erfolg oder Misserfolg der s.g. „Salvage Procedures“ nach fehlgeschlagener Osteosynthese proximaler Femurfrakturen entscheidet neben der Wahl des richtigen Operationszeitpunktes die Auswahl der adäquaten Operationsverfahren, denn auch beim Reeingriff ist die Erhaltung der Mobilität und eine möglichst kurze postoperative Immobilisation.
Grundvoraussetzung ist also ein kurzer, wenig belastender Eingriff mit sofortiger postoperativer Belastbarkeit $[5,19,26]$.

\section{Indikation und Verfahren}

Wie bei der Primärversorgung gelten auch bei der Reosteosynthese die von Stürmer 1993 formulierten Anforderungen an ein ideales Implantat für trochantäre Frakturen [25]:

Gewährleistung der Belastungsstabilität

- sichere Verankerung bei Osteoporose

- Fraktursinterung möglich

- Vermeidung der Implantatperforation in das Hüftgelenk

- Schonung der Vaskularisation

- einfache Implantationstechnik 
Tab. 1 Postoperative Komplikationen eine Rangfolge nach Smektala 1999 [24]

\begin{tabular}{lll}
$\begin{array}{l}\text { Häu- } \\
\text { figkeit }\end{array}$ & Komplikation & $\begin{array}{l}\text { Anteil } \\
(\%)\end{array}$ \\
\hline 1 & kardiovaskuläre & 6.58 \\
2 & pulmonale & 5.76 \\
3 & Wundheilungsstörung & 5.28 \\
4 & Harnwegsinfekt & 4.12 \\
5 & Serom/Hämatom & 4.10 \\
6 & Fieber $>38^{\circ}>2$ Tage & 2.30 \\
7 & Decubitus & 2.20 \\
8 & Thromboembolie & 2.06 \\
9 & Prothesenluxation & 1.38
\end{tabular}

Plattensysteme $\left(95^{\circ}, 120^{\circ}\right)$, DHS/DCS, intramedulläre Verfahren (proximaler Femurnagel, PFN, Gamma-Nagel etc.), Herniendoprothesen und als ultima ratio die Resektionsarthroplastik („Girdlestone Hüfte“) stellen mögliche Therapieoptionen nach fehlgeschlagener Osteosynthese proximaler Femurfrakturen dar.

Sie alle müssen der speziellen Situation nach fehlgeschlagener Osteosynthese einerseits und den biomechanischen Bedingungen andererseits am proximalen Femur als Ort einer exzentrischen Belastung Rechnung tragen (Abb.1-4: Fall $1-4)$.

Tab. 2 Komplikationen nach osteosynthetischer Frakturversorgung in Abhängigkeit von der Primärfraktur [22]

\begin{tabular}{lccc} 
Komplikation & Anteil (\%) & Schenkelhalsfraktur (\%) & $\begin{array}{l}\text { pertroch. Fraktur } \\
(\%)\end{array}$ \\
\hline Pseudarthrose & 34.9 & 37.1 & 45.4 \\
Femurkopfnekrose & 27.4 & 21.0 & 36.4 \\
posttraum. Coxarthrose & 11.7 & 12.1 & 9.1 \\
Pseudarthr. + Kopfnekrose & 11.0 & 12.9 & 9.1 \\
Pseudarth. + Material- & 4.8 & 4.8 & \\
dislok. & & & \\
Materialdislokation & 2.1 & 2.4 & \\
andere & 5.4 & 6.4 & \\
& & &
\end{tabular}

Das Therapiekonzept ist immer individuell zu erstellen und richtet sich nach der „5W“-Regel:

Lokalisation des „Fehlschlages“ (KopfHals-Fragment, Trochanter, Schaft):

$\square$ WO

Ursache des Fehlschlages (Versagen von Biologie, Implantat oder Operateur): $\square$ WARUM

Korrekte Auswahl der „Salvage-Procedure“ (Reosteosynthese, Endoprothese):

$\square$ WIE

Korrekte Auswahl des Implantates (Platte, Nagel):

$\square$ WOMIT

Optimaler Operationszeitpunkt:

$\square$ WANN
1. Vor einer Reosteosynthese muss beim jüngeren Patienten im Zweifelsfall die Vitalität des Hüftkopfes sowie das Defektausmaß im MRT verifiziert werden.

2. Je jünger ein Patient ist und je weiter nach subtrochantär gerichtet die Fraktur lokalisiert ist, umso eher sollte die Entscheidung zugunsten eines intramedullären Verfahrens fallen.

3. Je älter ein Patient ist und je weiter pertrochantär die Fraktur liegt, umso eher ist der Plattenosteosynthese der Vorzug zu geben

4. Je ausgedehnter der Defekt im KopfHals-Fragment und je schwerer die Osteoporose, umso eher sollte von eine Reosteosynthese zugunsten der Herniendoprothese abgesehen werden.
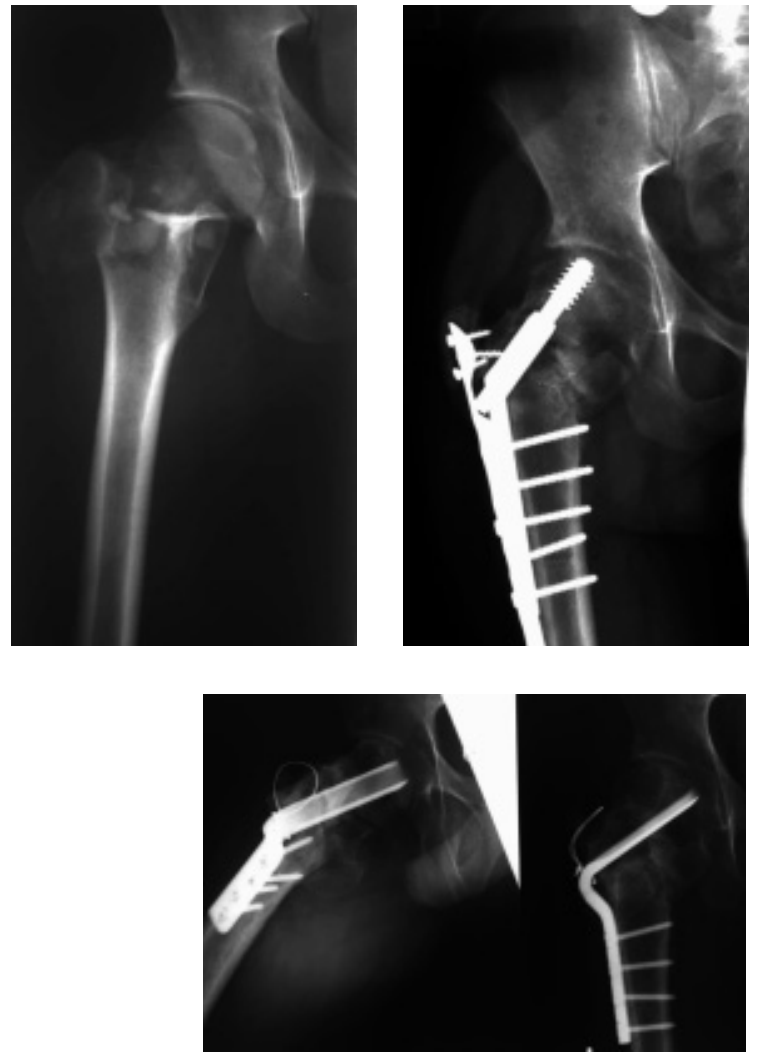

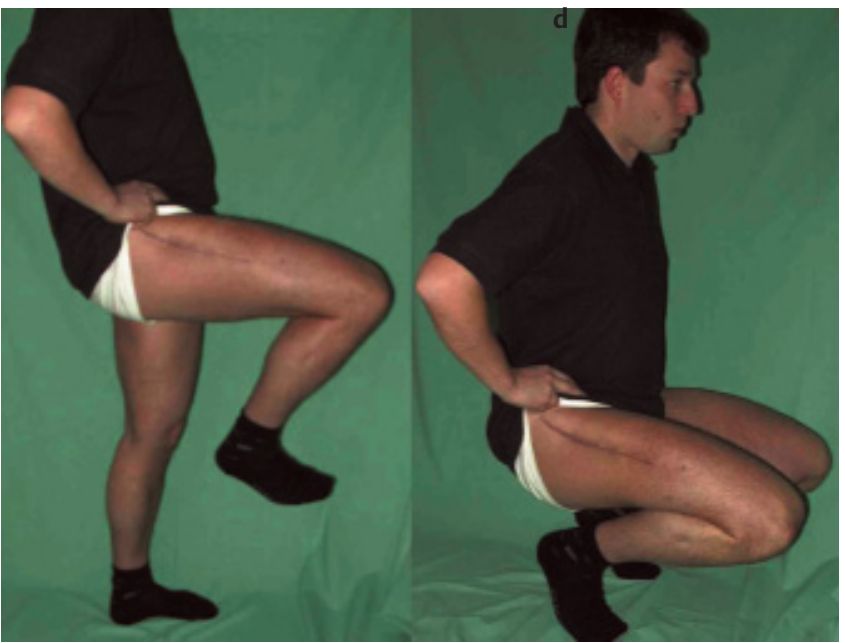

Abb.2 (a) 32-jähriger Mann mit pertrochantärer Oberschenkelfraktur. (b) Primärversorgung durch DHS und Trochanterabstützplatte, „Cut out" 6 Monate nach Unfall. (c) Reosteosynthese mit einer $135^{\circ}$-Klingenplatte 8 Monate nach Unfall. (d) klinisches Ergebnis 14 Monate nach Unfall. 

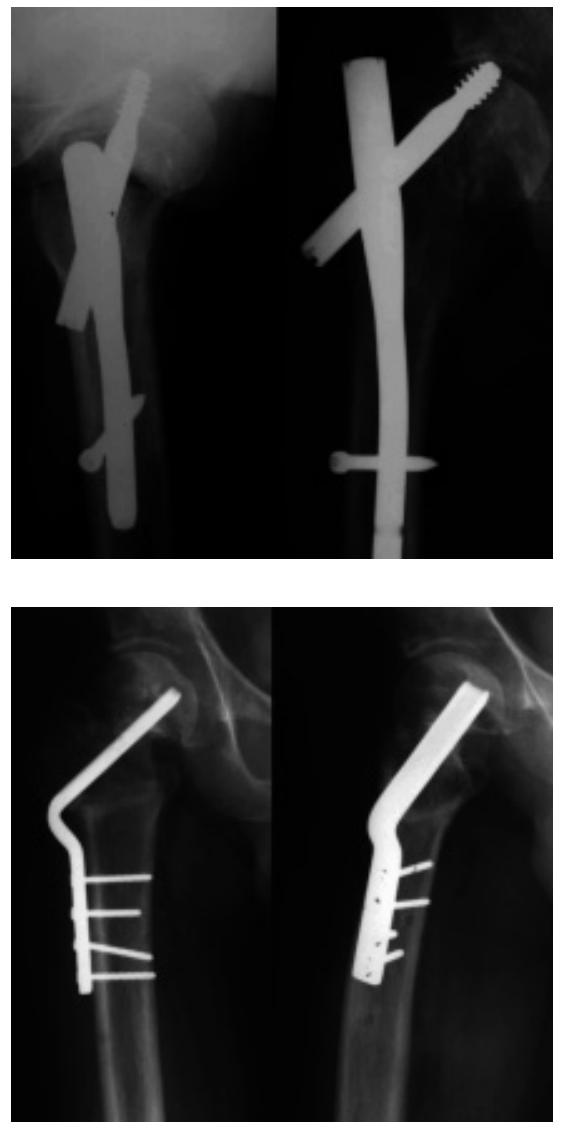

Abb.3 (a) 49-jähriger Mann mit pertrochantärer Oberschenkelfraktur, Primärversorgung mit Gamma-Nagel. „Cut out" nach 6 Monaten. (b) Reosteosynthese durch $135^{\circ}$-Klingenplatte 10 Monate nach Trauma.

5. In der Infektsituation ist auch beim alten Menschen dem zweizeitigen Vorgehen der Vorzug zu geben, d.h. zunächst Anstreben einer Girdlestonesituation, danach ggf. prothetische Versorgung.

6. Die Reosteosynthese mittels Winkelplatte verlangt hinsichtlich der Länge und Positionierung des Implantates im Femurkopf eine exakte Planung und Durchführung. Eine ausreichende Primärstabilität ist nur durch eine interfragmentäre Kompression (Plattenspanngerät) zu erreichen.

Neben der Wundinfektion und einer altersbedingt hohen Mortalität von bis zu $8 \%$ nach Versorgung einer pertrochantären Oberschenkelfraktur nimmt das biomechanische Therapieversagen eine zentrale Stellung ein [28]. So konnten beispielsweise Madsen et al. in einer vergleichenden Studie 1998 zeigen, dass es bei $9 \%$ der mit einer DHS und bei $4 \%$ der mit einem Gamma-Nagel versorgten Patienten zu einer sekundären Frakturdislokation oder zum „Cut-Out“ der Schenkel-
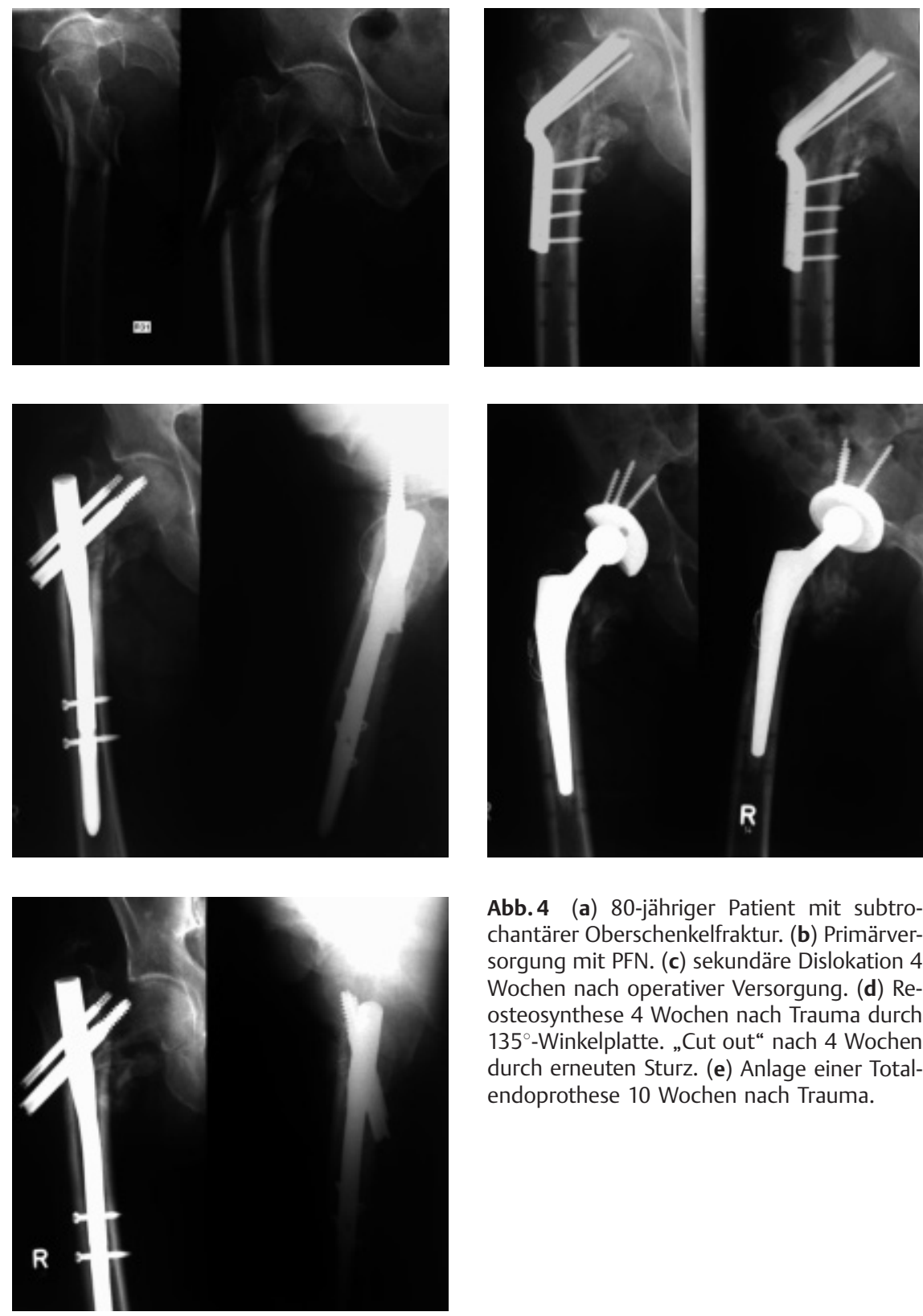

Abb.4 (a) 80-jähriger Patient mit subtrochantärer Oberschenkelfraktur. (b) Primärversorgung mit PFN. (c) sekundäre Dislokation 4 Wochen nach operativer Versorgung. (d) Reosteosynthese 4 Wochen nach Trauma durch $135^{\circ}$-Winkelplatte. „Cut out" nach 4 Wochen durch erneuten Sturz. (e) Anlage einer Totalendoprothese 10 Wochen nach Trauma.

halsschraube gekommen war. Noch höher lag diese Rate bei der ebenfalls nachuntersuchten Versorgung derartiger Frakturen mit einer „Hüftkompressionsschraube“. Hier kam es in 34\% zu den o.g. Komplikationen. Diese Tatsache führte in der DHSGruppe zu einer Reoperationsrate von $5,9 \%$, in der Gamma-Nagel-Gruppe von 8\% [17]. Parker konstatierte 1997 bei 74 mit einer DHS versorgten pertrochantären Femurfrakturen eine Dislokationsrate von 8\% [21]. Ebenfalls 1997 wiesen Lahoud et al. eine Dislokationsrate von $17,5 \%$ und eine „Non-Union-Rate“ von $10 \%$ bei Schraubenversorgung pertrochantärer Frakturen nach, bei den mit
Gamma-Nagel versorgten Frakturen lag

Eine Ursache für ein Therapieversagen ist die posttraumatisch gestörte Durchblutung des Kopf-Hals-Fragmentes. Auch wenn diese in ca. $75 \%$ erhalten bleibt und somit die Grundlage für eine komplikationslose Heilung nach Osteosynthese gegeben ist, treten in bis zu $30 \%$ Pseudarthrosen und in bis zu 26\% Femurkopfnekrosen auf $[20,22]$. Bei der Behandlung der Pseudarthrosen nach operativer Versorgung proximaler Femurfrakturen (i.e. Schenkelhals, per-/subtrochantär) sind die AO-Winkelplatten das Implantat der die Dislokationsrate bei 5\% [14]. 
Wahl, mit denen nach entsprechender präoperativer Planung eine Korrektur der Fehlstellung möglich ist $[9,23]$. Grundsätzlich gilt: Eine Veränderung in der Lastachse führt zu einer vermehrten Kompression im Pseudarthrosespalt unter Erhalt der Vitalität des proximalen Fragmentes. In Abhängigkeit vom Frakturverlauf kann beispielsweise eine intertrochantäre Valgisationsosteotomie vorgenommen werden, die mittels einer $120^{\circ}$ - oder $130^{\circ}$-Winkelplatte oder einer leicht aufgebogenen $95^{\circ}$-Kondylenplatte gehalten wird [11]. Marti et al. konnten mittels dieser Vorgehensweise 95\% der von ihnen behandelten Pseudarthrosen dieser Region zur Ausheilung bringen [18]. Ballmer et al. konnten 90\% zur Ausheilung bringen, wobei jedoch bei $30 \%$ der Patienten noch weitere Eingriffe notwendig waren [3].

Ein weiterer wesentlicher biologischer Faktor ist die Osteoporose, die auch bei korrekter Implantatwahl und - Platzierung zu einem Therapieversagen führen kann. Neuere Arbeiten zu diesem Thema zeigen, dass sich der Verlust an Knochenmasse mit zunehmendem Alter beschleunigt $[8,10]$.

Nicht nur die primär osteogenen Komplikationen spielen bei der Entscheidung für die Auswahl der weiteren Versorgung („Salvage-Procedure“) nach fehlgeschlagener Osteosynthese eine Rolle, sondern auch das Wissen um die Tatsache, dass die allgemeine Komplikationsrate bei derartigen Eingriffen mit dem Alter der Betroffenen korrelliert. Die Mortalität beträgt bereits bei der Primärversorgung für den 65-jährigen 10 bis 15\% [1]. Die erneute Operation ist mit einem neuerlichen Weichteilschaden und Minderung der lokalen Gewebsperfusion, dem Risiko einer weiteren Devitalisierung des Knochens und einer erhöhten Infektgefahr belastet. Die nochmalige postoperative Immobilisation erhöht das Risiko allgemeiner Komplikationen, wie kardiopulmonaler Dekompensation, Pneumonie etc. um ein Vielfaches (Tab.3) [22].

Primäres Ziel auch der Reoperation ist der Erhalt eines vitalen Femurkopfes. Insofern stellt die Reosteosynthese meist in Form einer Winkelplatte die Primärmaßnahme dar. Selten kann eine fehlgeschlagene intramedulläre Osteosynthese durch ein gleiches Verfahren behoben werden, da meist die Verankerung im Hüftkopf nicht gewährleistet ist. Dies trifft auch bei einer Vorosteosynthese mit einer dynamischen Hüftschraube
Tab.3 Darstellung der spezifischen intraoperativen sowie Frühkomplikationen $(<3$ Monate postop.) sowie der Spätkomplikationen (>3 Monate postoperativ) nach sekundärer Hüft-TEP-Implantation nach Perka [22].

\begin{tabular}{lc} 
Komplikationsform & $\begin{array}{c}\text { Anteil } \\
(\%)\end{array}$ \\
\hline intraop. Frühkomplikationen & 19.8 \\
Trochanterfrakturen, -fissuren & 6.9 \\
Femurschaftfrakturern, -fissuren & 5.8 \\
Ischiadikusparese & 1.4 \\
Wundheilungsstörungen & 4.8 \\
Luxation TEP & 1.4 \\
Spätkomplikationen & 4.1 \\
tiefe Protheseninfekte & 2.7 \\
Luxation TEP & 1.4
\end{tabular}

zu. Insofern kommt der $95^{\circ}$-Winkelplatte, die meist noch sehr gut im proximalen Femurbereich und Hüftkopf verankert werden kann, als Revisionsimplantat die Hauptbedeutung zu. Mit ihr lassen sich auch großstreckige Frakturbereiche im proximalen Femurbereich gut überbrücken. Limitierender Faktor ist hier die begrenzte Primärstabilität dieser Implantate, so dass hier die in der Regel durch die Patienten nicht eingehaltene Teilbelastung die Stabilität des Systemes an seine Grenzen führt.

Häufig verbleibt nach fehlgeschlagener Osteosynthese die Endoprothese als einziger Ausweg. Wesentliches Problem ist die gegenüber der elektiven Primärversorgung eklatant erhöhte Mortalität von $2-4 \%$ innerhalb der ersten 30 postoperativen Tage $[2,9,22]$, die innerhalb des ersten postoperativen Jahres auf ca. $8 \%$ ansteigt [20]. Die Mortalitätsrate dieser Eingriffe liegt aber deutlich unter der der notfallmäßigen primären Implantation bei hüftgelenksnaher Fraktur, die in der Literatur mit 5,7-15\% angegeben wird $[4,6,15]$. In einer Nachuntersuchung von 146 Patienten nach endoprothetischer Versorgung aufgrund einer fehlgeschlagenen Osteosynthese pertrochantärer Frakturen konnten Perka u. Mitarb. 2000 bei $85 \%$ der untersuchten Patienten gute bis exzellente Ergebnisse im Merle d'Aubigne Score nachweisen. Interessanterweise waren aber nur knapp 74\% der Patienten mit ihrem Operationsergebnis zufrieden. Die perioperative Mortalität wurde von diesen Autoren mit 2,7\% angegeben. Perka et al. kommen anhand ihrer Ergebnisse zu dem Schluss, dass die endoprothetische Versorgung die effektivste Form der „Salvage Procedure“ nach fehlgeschlagener hüftgelenknaher Osteosynthese darstellt [22]. Tabsh et al. wiesen 1997 nach, dass die Komplikationsrate der Hüft-Totalendoprothesen bei Patienten mit fehlgeschlagener Versorgung proximaler Femurfrakturen signifikant höher lag als bei Patienten ohne derartige Problematik. Interessanterweise zeigte sich jedoch im „HipScore“ zwei und mehr Jahre postoperativ kein signifikanter Unterschied zwischen Patienten mit primärer und sekundärer Hüft-Totalendoprothese [27]. Kompliziert eine Infektion die Situation zusätzlich, ist häufig die Entfernung des Osteosynthesematerials und die Anlage einer „Girdlestone-Situation“ der einzige Ausweg. Abhängig vom Allgemeinzustand und dem Mobilisationsgrad vor der Verletzung kann nach Infektsanierung die Implantation einer Hüftendoprothese erwogen werden.

\section{Fazit}

Die proximale Femurfraktur stellt die häufigste Fraktur des älteren Menschen dar, mit weiter steigender Tendenz. Die schnellstmögliche optimale, d.h. belastungsstabile Versorgung wird somit zum zentralen Problem für den behandelnden Chirurg. Das Konzept der Primärversorgung beinhaltet neben einem den Gesamtorganismus schonenden operativen Vorgehen die konsequente Minimierung der häufigsten Frühkomplikationen durch den Einsatz von niedermolekularem Heparin (Thromboseprophylaxe), intraoperativer Single-Shot-Antibiotika-Prophylaxe (Vermeidung von lokalen Infekten), Frühmobilisation unter krankengymnastischer Anleitung (Atemtraining, Gangschule) aber auch der Iniziierung einer adäquaten Nachbetreuung („Essen auf Rädern“, Sozialdienste, Anschlussheilbehandlungen). Die im weiteren Verlauf auftretenden Komplikationen bestehen in erster Linie aus Knochenbiologie oder implantatassoziierten Störungen, die in der Regel eine Reoperation unumgänglich machen. Im Fall der Reoperation müssen klare Konzepte hinsichtlich Biomechanik angewandt werden, um weitere Probleme zu verhindern.

\section{Literatur}

${ }^{1}$ Albright JP, Weinstein SL. Treatment for Fixation Complications. Arch Surg /Vol 1975; 110: $30-36$

2 Babst R, Jenny H, Morschner E. Die Behandlung der infizierten Hüftgelenksarthroplastik. Orthopäde 1989; 18: 517 - 526

${ }^{3}$ Ballmer FT, Ballmer PM, Mast JW, Ganz R. Results of repositioning osteotomies in de- 
layed healing or pseudarthrosis of the proximal femur. Unfallchirurg Oct 1992; 95 (10): $511-517$

${ }^{4}$ D'Arcy J, Devas M. Treatment of fractures of the femoral neck by replacement with the Thompson Prosthesis. J Bone Joint Surg 1976; 58-B: 279-286

${ }^{5}$ Degreif J, Hammer M, Wenda K, Ritter G. Langzeitergebnisse nach Versorgung medialer Schenkelhalsfrakturen mit Keramikkopfprothesen. Unfallchirurg 1996; 99: 7444

${ }^{6}$ Diercks AL, Hollander H. Mo(o)re or less prostheses: retrospective study of 166 dislocated femoral neck fractures treated by the Moore Prosthesis. Neth J Surg 1985; 37: 11 15

${ }^{7}$ Dzupa V, Bartonicek J, Skala-Rosenbaum J, Prikazsky V. Mortality in patients with proximal femoral fractures during the first year after injury. Acta Chir Orthop Traumatol Cech 2002; 69 (1): 39-44

${ }^{8}$ Ensrud KE, Palermo L, Black DM, Cauley J, Jergas M, Orwoll ES, Nevitt MC, Fox KM Cummings SR. Hip and calcaneal bone loss increases with advancing age: longitudinal results from the Study of Osteoporotic Fractures. J Bone Miner Res 1995; 10: 1778 - 1787

${ }^{9}$ Halder SC. The Gamma nail for peritrochanteric fractures. J Bone Joint Surg Br 1992; 74 :340

${ }^{10}$ Jones G, Nguyen T, Sambrook P, Eisman JA. Progressive loss of bone in the femoral neck of elderly people: longitudinal findings from the Dubbo Osteoporosis Epidemiology Study. B Med J 1994; 309: 691 -695

${ }^{11}$ Josten Ch, Verheyden P. Pertrochantäre Pseudarthrosen. Materialfehler - technische Fehler - naturgegeben. Chirurg 1999; 70 : $1209-1215$

${ }^{12}$ Kaukonen JP, Kemppainen E, Mäkijärvi J, Tuominen T. One dose cefuroxime prophylaxis in Surgery of the hip. Ann Chir Gyn 1995; 84: $417-419$
${ }^{13}$ Kuokkanen H. Operative treatment of femoral neck fractures. A clinical study. Academic dissertation, University of Helsinki (1992)

${ }^{14}$ Lahoud JC, Asselineau A, Salengro S, Molina V, Bombart M. Sub-trochanteric fractures. A comparative study between gamma nail and angular osteosynthesis with lateral cortica support. Rev Chir Orthop Reparatrice Appar Mot 1997; 83 (4): 335-342

${ }^{15}$ Lausten GS, Vedel P, Nielsen P. Fractures of the femoral neck treated by bipolar prostheses. Clin Orthop 1987; 218: 63-67

${ }^{16}$ Lu-Yao G, Keller RB, Littenberg B, Wennberg JE. Outcomes after displaced fractures of the femoral neck. A meta-analysis of one hundred and six published reports. J Bone Joint Surg 1994; 76a: 15-25

${ }^{17}$ Madsen JE, Naess L, Aune AK, Alho A, Ekeland A, Stromsoe K. Dynamic hip screw with trochanteric stabilisation plate in the treatment of unstable proximal femoral fractures: a comparative study with Gamma nail and compression hip screw. J Orthop Trauma May 1998; 12 (4): 241 - 248

${ }^{18}$ Marti R, Raaymakers ELFB, Nolte P, Besselaar PP. Pseudarthrosen am proximalen Femur. Orthopäde 1996; 25: 454

${ }^{19}$ Niebuhr H, Nahrstedt U, Brünning M, Rückert K. Variokopfprothese in der Behandlung der Schenkelhals- und schenkelhalsnahen Fraktur. Unfallchirurgie 1991; 17: 146 151

${ }^{20}$ Nilsson L, Strömquist B, Thorngren KG. Secondary Arthroplasty For Complications Of Femoral Neck Fracture J Bone Joint Surg [Br] 1989; 71-B: 777-781

21 Parker MJ, Dutta BK, Sivaji C, Pryor GA. Subtrochanteric fractures of the femur. Injury Mar 28 (2): 91 - 95

${ }^{22}$ Perka C, Ludwig R, Stern S. Die totalendoprothetische Versorgung nach fehlgeschlagener Osteosynthese einer hüftgelenksnahen Fraktur. Z Orthop 2000; 138: 39-45
${ }^{23}$ Sarathy MP, Madhavan P, Ravichandran KM. Nonunion of intertrochanteric fractures of the femur. J Bone Joint Surg Br 1995; 77: 90

${ }^{24}$ Smektala R, Wenning M, Ekkernkamp A. Schenkelhalsfraktur: Analyse der Ergebnisse externer Qualitätssicherung. Chirurg 1999; 70: $1330-1339$

${ }^{25}$ Stürmer KM. et al. Wandel bei der Osteosynthese pertrochantärer und subtrochantärer Femurfrakturen. H.z.D. Unfallchir, H 232 K.E. Rehm (Hrsg) Springer Berlin Heidelberg: 1993; 99-121

${ }^{26}$ Swiontkowski MF. Femoral neck fractures. In: Browner BD., Jupiter JB., Levine AM., Trafton PG. (eds) Skeletal trauma. Saunders, Philadelphia London, 1992; pp 1384-1442

${ }^{27}$ Tabsh I, Waddell JP, Mortonj. Total hip arthroplasty for complications of proximal femoral fractures. J Orthop Trauma Apr 1997; 11 (3): $166-169$

${ }^{28}$ Vajanto I, Kuokkanen H, Niskanen R, Haapala J, Korkala O. Complications after treatment of proximal femoral fractures. Ann Chir Gynaecol 1998; 87 (1): 49-52

\section{Prof. Dr. med. Christoph Josten} Direktor

Dr. med. Andreas Heinrich Tiemann Oberarzt der Chirurgischen Klinik III

Universität Leipzig AöR

Klinik und Poliklinik für Unfallund Wiederherstellungschirurgie Liebigstr. 20 a

D-4103 Leipzig 